\title{
The Transportation Problem Under Probability and Fuzzy Uncertainties
}

\author{
U. Deva ${ }^{a}$ A. Sultana ${ }^{b}$ and N. K. Mitra ${ }^{d}$ \\ ${ }^{a}$ Department of Mathematics, Jagannath University, ${ }^{b}$ Department of Mathematics, Jahangirnagar University, \\ Savar and. ${ }^{c}$ Department of Mathematics, Bangladesh University of Business and Technology, Dhaka.
}

\begin{abstract}
The paper presents the more developed approach for the transportation problem solution in the case of fuzzy co-efficients. The implementation of fuzzy extension of general simplex method is used to realize the numerical fuzzy optimization with fuzzy constraints. The proposed fuzzy numerical method is based on the practical system of the Pioneer Stefan Chanas idea to consider the fuzzy numbers in the probabiilistic sense. The solution of the problem is more generalized based upon the distributor's benefit maximization.
\end{abstract}

Key words: Transportation problem, Fuzzy co-efficients, Fuzzy optimization, Transporting costs, Practical system.

\section{Introduction}

The distributor's decisions optimization can be reformulated as the generalization of classical transportation problem. Conventional transportation problem is the special type of linear programming problem in which we used special mathematical structure of restrictions. In classical transporting costs from $\mathrm{K}$ wholesalers to the $\mathrm{L}$ consumers are minimuzed.

In 1979, Isermann (Isermann, 1979) introduced algorithm for solving this problem. The Ringuest and Rinks (Dymowa and Dolata) proposed two iterative algorithms for solving linear, multicriterial transportation problem. Similar solution proposed in 1992 by A. K. BIT (Bit et. al., 1992).

The different effective algorithms were worked out for this transportation problem but parameters for task described in form of real numbers. Nevertheless, such conditions are fulfilled almost never because of on natural uncertainties in which we met real world problems. For example, it is hard to define stable cost of specified rout. In 1999, S.K. DAS (Das et. al., 1999), this problem was solved in case of interval uncertainty of transporting costs.

In 1993 \& 1998 works by S. Chanas and D. Kuchta (Chanas et. al., 1993, Chanas and Kuchta, 1998), the result based on interval and fuzzy Co-efficient had been elaborated. The further development of this approach introduced by F. WAIEL in 2001 (Waiel and ABD, 2001).
All the above mentioned works introduce the restrictions in a form of membership function. This allows to transform the initial fuzzy linear programming problem into the net of usual linear programming tasks by use of well defined analytic procedures. However in practice the membership functions, which describes uncertain parameters of used models can have the considerable complicated forms. In such cases, the numerical approach is needed.

The main technical problem when constructing the numerical fuzzy optimization algorithm is to compare the fuzzy values. To decide this problem, we use the approach proposed by P. SEWASTIANOW in 2002 \& 2003 (Sewastianow et. al., 2002, Sewastianow and Gog, 2003) and well described by P. SEWASTIANOW \& M. JONCZYK (Sewastianow and Jonczyk), which is -level representation of fuzzy numbers and probability estimation of the fact that given interval is greater than equal an other interval. We note that probabilistic method was used only to infer the set of formulae for deterministic quantitative estimation of intervals inequality or equality.

The method allows to compare the interval and real number and to take into account the widths of intervals ordered.

The proposed method allows to accomplish the direct fuzzy extension of classical numerical simplex method with its

\footnotetext{
* Corresponding author: E-mail:
} 
implementation using tools of object orented programming (Waiel and ABD, 2001).

\section{Preliminaries}

\section{Definition 2.1 : Optimization}

A linear programming requires the optimization of a desired object such as minimization of cost or maximization of profit or achievement of desired performance utility through the system under constraints.

\section{Definition 2.2: Fuzzy Optimization}

(Zimmermann H.J., 1996). In the following case a fuzzy decision model is employed as optimization criterion for a search algorithm. It proved to better reflect human judgment in a scheduling problem when due dates were tight.

The standard operation research approach is to model the tardiness of a schedule as the objective function to be minimized, defined by:

* the number of late jobs

* the sum of delays of all late jobs

* the delay of the latest job

* the weighted sum of late job (penalty cost approach)

Definition 2.3 : Fuzzy Approach to the transportation problem

(Zimmermann H.J., 1996) The analysis of "fuzzy counterparts" of linear programming problems of some special structure, for example problems of flows in networks, transportation problems, and so on, appears to be an interesting task. The following model considers a transportation problem with fuzzy supply values of the suppliers and with fuzzy demand values of the receivers. For the solution of the problem parametric programming is used.

Model. 1:

$$
\begin{array}{ll}
\text { Minimize } & C=\sum_{i=1}^{m} \sum_{i=1}^{n} C_{i j} \mathrm{X}_{\mathrm{ij}} \\
\text { such that } & \sum_{j=1}^{n} X_{i j} \cong \widetilde{a}_{i}, \quad=1,2, \ldots \ldots, \mathrm{m} \\
& \sum_{j=1}^{m} X_{i j} \cong \widetilde{b}_{j}, \mathrm{j}=1,2, \ldots . ., \mathrm{n} \\
& \mathrm{X}_{\mathrm{ij}} \geq 0, \mathrm{i}=1,2, \ldots \ldots . . \mathrm{m} ; \mathrm{j}=1,2, \ldots \ldots ., \mathrm{n}
\end{array}
$$

$\widetilde{a}_{j}$, and $\widetilde{b}_{j}$ lenote non-negative fuzzy numbers of trapezoidal form. The value of $\mu_{\tilde{a}}\left(\sum_{j} X_{i j}\right)\left(\mu_{\tilde{b}}\left(\sum_{j} X_{i j}\right)\right)$ s interpreted as a feasibility degree of the solution w. r. t. the i-th (jth) constraint in model 1 .

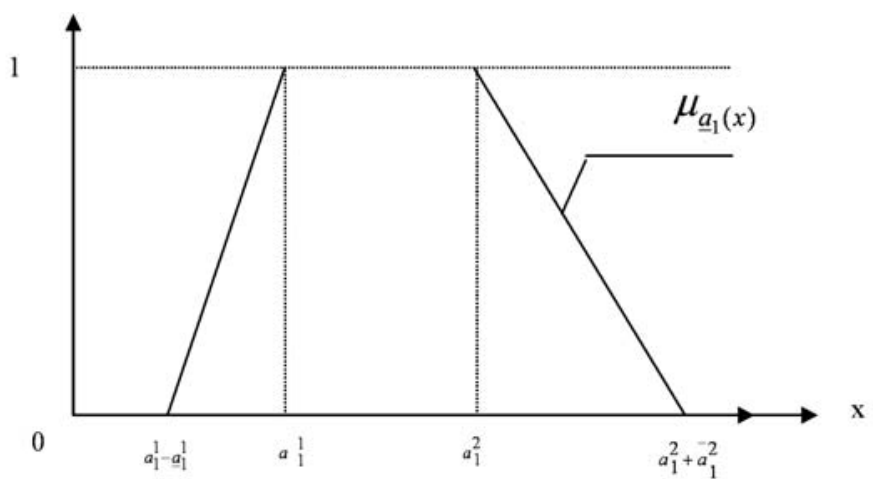

Fig. 1: The trapezoidal form of a fuzzy number.

$$
\bar{a}_{1}=\left(a_{1}^{1}, \underline{a}_{1}^{1} a_{1}^{2}, \bar{a}_{1}^{2}\right)
$$

\section{Definition 2.4 Simplex method}

Simplex method also called simplex technique or simplex algorithm was developed by G. B. Dantzig (in 1947), an American mathematician. The simplex method provides an excellent of the algorithms (iterative solution procedures) that are so prevalent in operations research work.

\section{Definition 2.5 Uncertainty method:}

(Zimmermann H.J., 1996) There are three main reasons for the use of fuzzy set theory in expert systems : (i) The interfaces of the expert system on the expert side as well as on the user side are with human beings. Therefore communication in a 'natural' way seems to be the most appropriate; and 'natural' means, generally, in the language of the expert or user.

(ii) The knowledge base of an expert system is a repository of human knowledge; and since much of human. Knowledge is imprecise in nature it is usually the case that the knowledge base of an expert system is a collection of rules and facts which, for the most part are neither totally certain, nor totally consistent.

(iii) As a consequence of what has been said in point two the 'management of uncertainty' plays a particularly important role. Uncertainty of information in the knowledge base indices uncertainty in the conclusions. 
Definition 2.6 Probability of a Fuzzy Evant as a fuzzy set (Zimmermann H.J., 1996) Let $\widetilde{A}=\left\{\left(\mathrm{x}, \mu_{\overline{\mathrm{A}}}(x)\right) \mid x \in X\right\}$ be a fuzzy set representino a filzzy event. The degree of membership of element $x_{i} \in \widetilde{A}$ is denoted by $\mu_{\widetilde{A}}\left(\mathrm{x}_{\mathrm{i}}\right) . \alpha-$ level sets or $\alpha$-cuts such as already defined shall be denoted by $A_{\alpha}$.

Yagen [1979, 1987] suggests that it is quite naturo1 to define the probability of an $\alpha$-level sets as $\mathrm{P}\left(\mathrm{A}_{\alpha}\right)=\sum_{x \in A_{i j}} \mathrm{P}(\mathrm{x})$. On the basis of this the probability of a fuzzy event 1s defined as follows [Yager 1984].

\section{Definition 2.6.1}

Let $\mathrm{A}_{\alpha}$ be the $\alpha$-level set of a fuzzy set $\tilde{A}$ representing a fuzzy event. When the probability of a fuzzy event $\tilde{A}$ can be defined as $P_{Y}(\widetilde{A})=\left\{\left(P\left(A_{\alpha}\right), \alpha\right) \mid \alpha \in[0,1]\right\} \quad$ with the interpretation "the probability of at least an $\alpha$ degree of satisfaction to the condition $\widetilde{A}$."

\section{Definition 2.7 Fuzzy Linear Programming}

(Zimmermann H. J., 1996) Linear programming models shall be considered as a special kind of decision model : the decision space is defined by the constraints; the "goal" (utility function) is defined by the objective function; and the type of decision is decision making of linear programming can be stated as

maximize $\mathrm{f}(\mathrm{x})=\mathrm{C}^{\mathrm{T}} \mathrm{x}$

such that $\mathrm{Ax} \leq \mathrm{b}$

$$
\mathrm{x} \geq 0
$$

with $c, x \in R^{n}, b \in R^{m}, A \in R^{m \times n}$.

The Method's description of linear programming frequency distribution and fuzzy number

In the proposed method we not only minimize the transportation costs but in addition we maximize the distributor's profits with the same manner.
Let $\mathrm{a}_{\mathrm{i}}(\mathrm{i}=1$ to $\mathrm{K})$, be the maximal quantities of goods that can be proposed by wholesalers and $b_{j}(j=1$ to $L)$, be the maximal good requirements of consumers. The distributor must buy at least $p_{i}$ good units at price of $t_{i}$ monetary units for unit of good from each ith wholesaler and to sell at least $\mathrm{q}_{\mathrm{j}}$ good units at price of $\mathrm{s}_{\mathrm{j}}$ monetary units for unit of good to each jth consumer. The total transportation cost of delivering good unit from ith wholesaler to jth consumer is $\mathrm{c}_{\mathrm{ij}}$.

For distributor, the reduced prices are $\mathrm{m}_{\mathrm{i}}$ if he or she buy the greater quantities of good then stipulated in contract quantities $p_{i}$ and also the reduced prices $n_{j}$ for consumers if they buy the good quantities grater than contracted $\mathrm{q}_{\mathrm{j}}$. The problem is to find the optimal good quantities $x_{i j}(i=1$ to $K ; j=$ 1 to $\mathrm{L}$ ) delivering from ith wholesaler to jth consumer maximizing the distributor's total benefit B under restrictions. All above mentioned parameters are fuzzy ones, resulting optimization formulated as :

Maximize $B=\sum_{i=1}^{K} \sum_{j=1}^{L}\left(Z_{i j} * x_{i j}\right)$

Subject to constraints

$$
\left.\begin{array}{l}
\sum_{j=1}^{L} x_{i j} \leq a_{i},(i=1 \text { to } \mathrm{K}) \\
\sum_{i=1}^{K} x_{i j} \leq b_{j},(j=1 \text { to } \mathrm{L})
\end{array}\right\}
$$

Where $Z_{i j}=n_{j}-m_{i}-c_{i j}(i=1$ to $K ; j=1$ to $L)$ and $B, Z_{i j}$, a, $\mathrm{b}, \mathrm{p}, \mathrm{q}$ are fuzzy values.

The distributor contracts with $\mathrm{K}$ wholesalers and L consumers (Fig. 1).

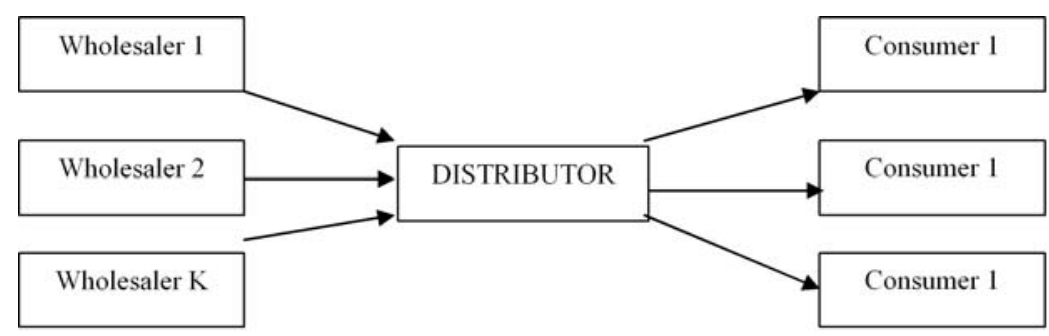

Fig. 1. The distributor's activity 
To decide the problem (1) - (3), the numerical method based on the $\alpha$-cut representation of fuzzy numbers and probabilistic approach to the interval and fuzzy interval comparison has been elaborated.

The direct fuzzy extension of usual simplex method is used. The use of object programming tools makes it possible to get the results of fuzzy optimization. $\mathrm{i}$, e. $\mathrm{x}_{\mathrm{ij}}$, in the form of fuzzy numbers.

To estimate the effectiveness of method proposed the results of fuzzy optimization were compared with thouse obtained from (1)-(3) when all the uncertain parameters were considered as normally distributed random values. In the last case all the parameters in (1) - (3) were considered as real numbers.

To make the results using the fuzzy and probability approaches comparable the simple special method for transformation frequency distributions into fuzzy numbers without lost of useful information was used to achieve the comparability of uncertain initial data in fuzzy and random cases.

In practice, we often have a problem with different precisions of representation the uncertain data we use. For instance, one part of parameters used can be represented in the trapezoid fuzzy numbers form on basis of the expert's opinions and at the same time, the other part of them can have the form of the histogram or frequency distributions of considerable complicated form we got as a result of statistical analysis.

In these cases, the methodologically correct approach is to transform all the uncertain data available to the form of smallest certain level we met in our task. Thus, we have to transform the data represented in form of frequency distributions or histogram to the membership functions of fuzzy numbers.

To present the initial data in fuzzy number form at first we should apply an algorithm, which builds the membership function on basis of frequency distribution, if such exists, or directly using histogram.

In the simplest case of normal frequency distributions, they can be exhaustively described be their averages $\mathrm{d}$ and stan- dard deviations $\sigma$. In the more complicated situations it seems better to use directly the histograms.

That is why, we use the numerical algorithm which allows us to transform the frequency distribution or histogram to trapezoidal fuzzy number.

As the illustration, let us consider the reduction of frequency distribution (Fig. 2) to the fuzzy number.

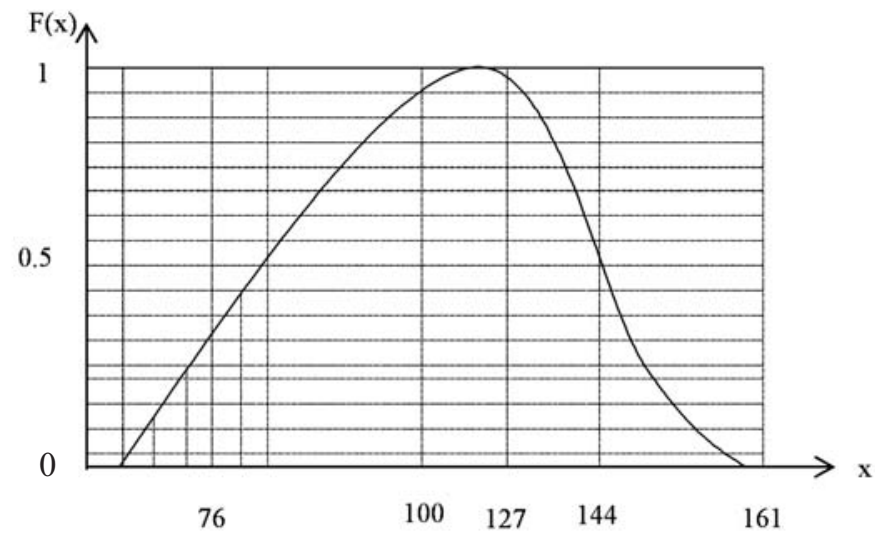

Fig. 2: Frequency distribution to be transformed

We have to accomplish the following steps of algorithm.

\section{Step 1.}

In the interval within the smallest value $\mathrm{x}_{\min }$ (in our example $\left.\mathrm{x}_{\min }=60\right)$ and maximum value $\left(\mathrm{x}_{\max }=161\right)$, we define the function $\mathrm{F}\left(\mathrm{x}_{\mathrm{i}}\right)$ as surface are under the curve (Fig. 2) from $\mathrm{x}_{\min }$. to current the $\mathrm{x}_{\mathrm{i}}$. As a result, we get cumulate function show on (Fig. 3). It is easy to see that function $F(x)$ is factually, the probability of $\mathrm{x}<\mathrm{x}_{\mathrm{i}}$.

\section{Step. 2.}

Using obtained cumulate function $\mathrm{F}(\mathrm{x})$ we ask the decision makers (experts) for the four values $\mathrm{F}\left(\mathrm{x}_{\mathrm{i}}\right)(\mathrm{i}=0,1,2,3)$ which define the mapping of $\mathrm{F}(\mathrm{x})$ on $\mathrm{X}$ in such a way that they provide the bottom and upper $\alpha$ - levels of trapezoidal fuzzy number. In our example (Fig. 3), the intervals [105, $115]$ and $[88,130]$ are in essence the $30 \%$ and $90 \%$ probability confidence intervals. 


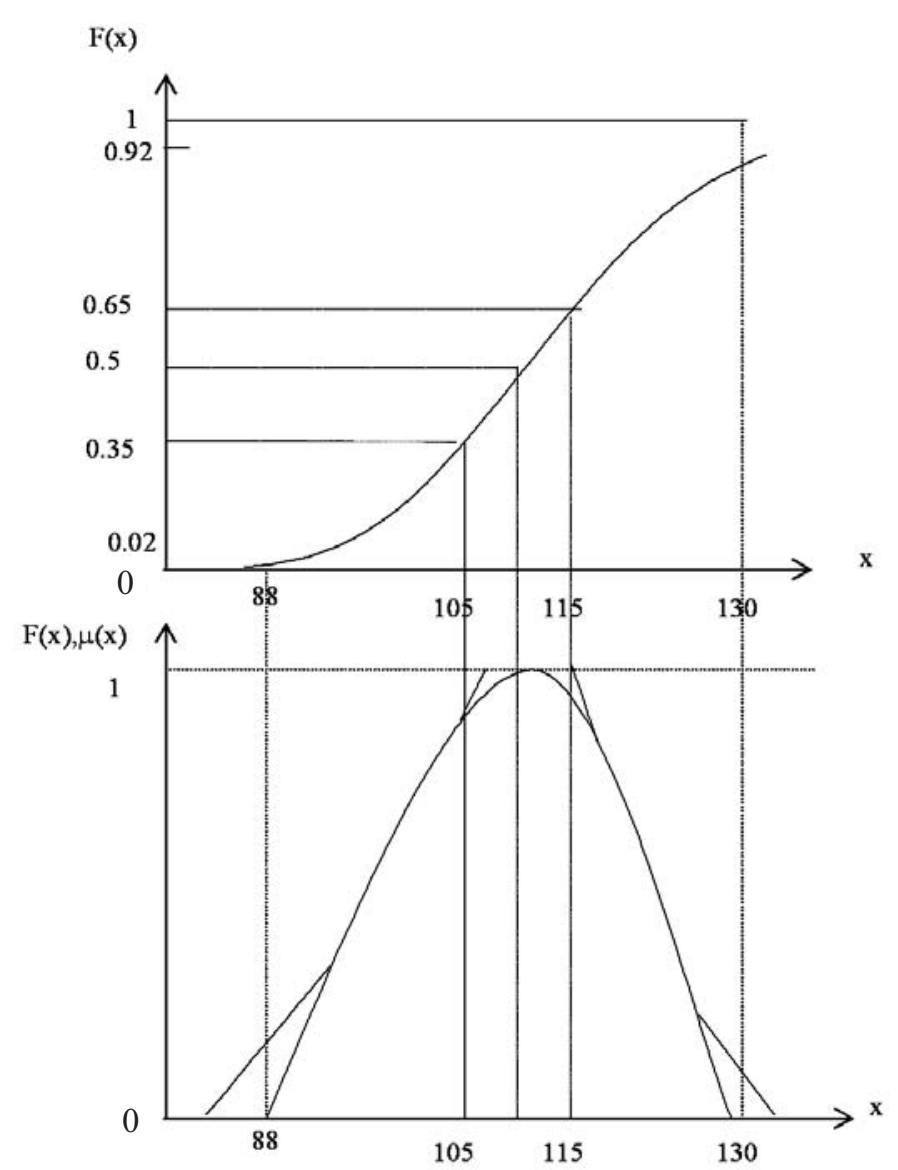

Fig. 3: I he transtormation of cumulate function to a fuzzy number

As a result we get the trapezoidal fuzzy interval represented in our example by quadruple $[88,105,115,130]$.

It is easy to see that transformation accuracy depends only on the expert's opinion about suitability and correctness of upper and button confidence intervals chosen.

It is worthy to note that the main advantage of presented method is that it can be successfully used in both cases; when we have the initial data in a form of frequency distribution function and in a form of rough histogram.

The described method allows us to represent all the uncertain data in uniform way as the trapezoid fuzzy intervals.

The elaborated method of fuzzy programming problem (1) (3) solution is realized performing all fuzzy numbers as the sets of $\alpha$-cuts. In fact, it reduces fuzzy problem into the set of crisp interval optimization tasks.

The final solution has been obtained numerically with using probabilistic approach to interval comparison. The interval arithmetic rules needs were realized with a help of object oriented programming tools.

The standard Monte-Caro procedure was used for the realization of probability approach to the description of uncertain parameters of the optimization task (1)-(3). In fact, for each randomly selected set of real valued parameters of task (1)-(3). We solve the usual linear programming problem.

\section{Numerical Example}

To compare the results of fuzzy programming with those obtained when using the Monte-Carlo method, all the uncertain parameters previously were performed by Gaussian frequency distribution. The averages of them are presented in Table I. For simplicity, all the standard deviations were accepted as equal to $10 \mathrm{i}$. $\mathrm{m}$.

The results we get with using of fuzzy optimization method and Monte-Carlo method (usual linear programming with real valued but random parameters) are presented in Fig. 4 to Fig. 8 for the case $\mathrm{K}=\mathrm{L}=3$, where the final frequency distributions $\mathrm{F}$ are drown by dotted lines, fuzzy numbers $\mu$ are drawn by continuous lines.

Table I: Average values of Gaussian distributions of uncertain parameters

\begin{tabular}{l|l|l|l|l|l|l|l}
\hline $\mathrm{a}_{1}=360$ & $\mathrm{~b}_{1}=310$ & $\mathrm{p}_{1}=340$ & $\mathrm{q}_{1}=290$ & $\mathrm{t}_{1}=500$ & $\mathrm{~s}_{1}=900$ & $\mathrm{~m}_{1}=490$ & $\mathrm{n}_{1}=890$ \\
$\mathrm{a}_{2}=360$ & $\mathrm{~b}_{2}=410$ & $\mathrm{p}_{2}=340$ & $\mathrm{q}_{2}=390$ & $\mathrm{t}_{2}=391$ & $\mathrm{~s}_{2}=391$ & $\mathrm{~m}_{2}=380$ & $\mathrm{n}_{2}=1000$ \\
$\mathrm{a}_{3}=510$ & $\mathrm{~b}_{3}=510$ & $\mathrm{p}_{3}=490$ & $\mathrm{q}_{3}=490$ & $\mathrm{t}_{3}=481$ & $\mathrm{~s}_{3}=481$ & $\mathrm{~m}_{3}=470$ & $\mathrm{n}_{3}=1080$ \\
$\mathrm{c}_{11}=90$ & $\mathrm{c}_{12}=20$ & $\mathrm{c}_{13}=90$ & & & & \\
$\mathrm{c}_{21}=100$ & $\mathrm{c}_{22}=26$ & $\mathrm{c}_{23}=395$ & & & & & \\
$\mathrm{c}_{31}=110$ & $\mathrm{c}_{32}=138$ & $\mathrm{c}_{33}=01$ & &
\end{tabular}


Fuzzy optimization has been formulated as :

$$
\begin{aligned}
\operatorname{maximize} B= & \sum_{i=1}^{3} \sum_{j=1}^{3}\left(Z_{i j} \times x_{i j}\right) \\
= & \sum_{i=1}^{3}\left(Z_{i 1} x_{i 1}+Z_{i 2} x_{i 2}+Z_{i 3} x_{i 3}\right) \\
& \sum_{j=1}^{3} x_{i j} \leq a_{i}, \quad \sum_{i=1}^{3} x_{i j} \leq b_{j} \\
& \sum_{j=1}^{3} x_{i j} \geq p_{i}, \quad \sum_{i=1}^{3} x_{i j} \leq q_{j}
\end{aligned}
$$

Maximize $\mathrm{B}=310 \mathrm{x}_{11}+410 \mathrm{x}_{21}+310 \mathrm{x}_{31}+490 \mathrm{x}_{12}+594 \mathrm{x}_{22}$ $+392 x_{32}+500 x_{13}+305 x_{23}+609 x_{33}$.

$$
\begin{aligned}
& \text { Subject to } x_{11}+x_{12}+x_{13} \leq 360 \\
& x_{21}+x_{22}+x_{23} \leq 360 \\
& x_{31}+x_{32}+x_{33} \leq 510 \\
& x_{11}+x_{21}+x_{31} \leq 310 \\
& \mathrm{x}_{12}+\mathrm{x}_{22}+\mathrm{x}_{32} \leq 410 \\
& \mathrm{x}_{13}+\mathrm{x}_{23}+\mathrm{x}_{33} \leq 510 \\
& \mathrm{x}_{11}+\mathrm{x}_{12}+\mathrm{x}_{13} \geq 340 \\
& \mathrm{x}_{21}+\mathrm{x}_{22}+\mathrm{x}_{23} \geq 340 \\
& x_{31}+x_{32}+x_{33} \geq 490 \\
& x_{11}+x_{21}+x_{31} \geq 290 \\
& x_{12}+x_{22}+x_{32} \geq 390 \\
& x_{13}+x_{23}+x_{33} \geq 490 \\
& \bar{F}\left(\mathrm{X}_{1}\right\}, \ldots\left\{\mathrm{X}_{\mathrm{f}}\right\}
\end{aligned}
$$

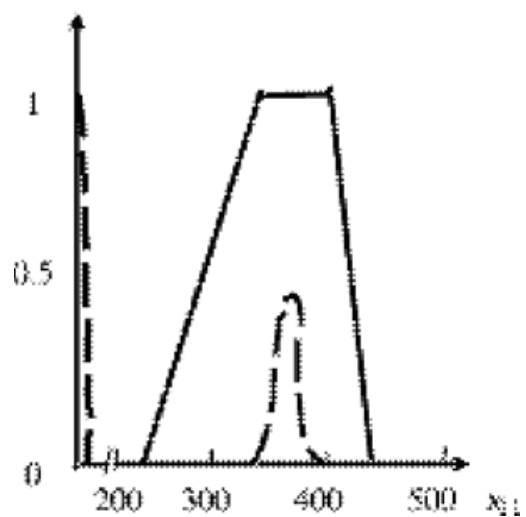

Fig. 4: Frequency distribution $F$ and fuzzy number $\mu$ for optimized $x_{11}$.

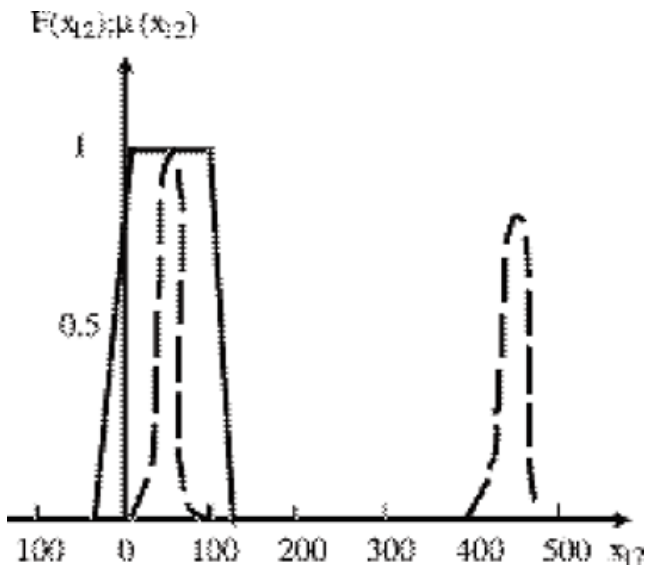

Fig. 5: Frequency distribution $F$ and fuzzy number $\mu$ for optimized $x_{12}$.

$$
\text { F(xys) }
$$

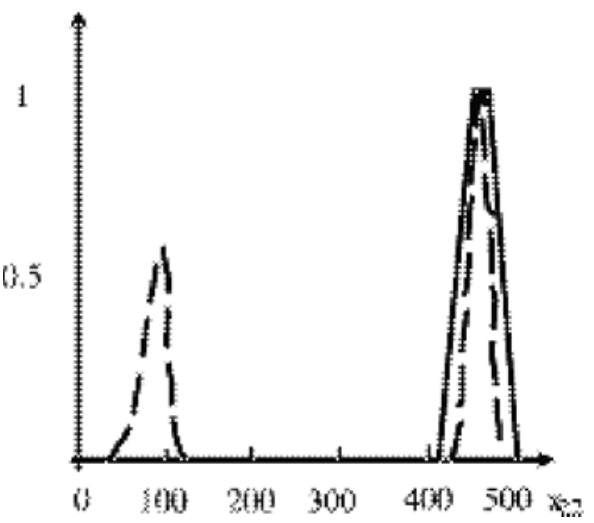

Fig. 6: Frequency distribution $F$ and fuzzy number $\mu$ for optimized $x_{22}$.

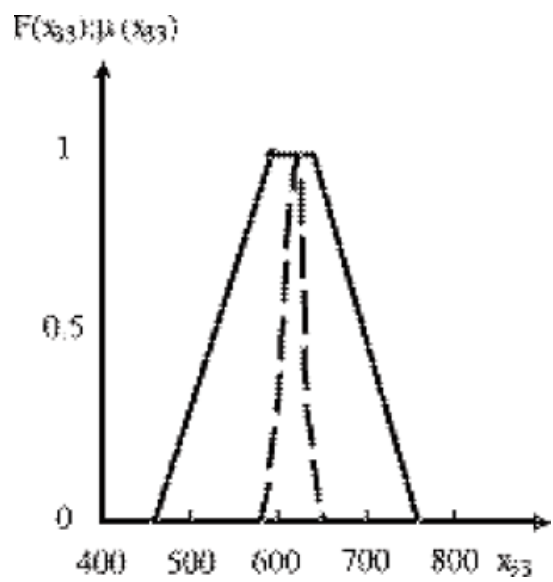

Fig. 7: Frequency distribution $F$ and fuzzy number $\mu$ for optimized $x_{33}$. 


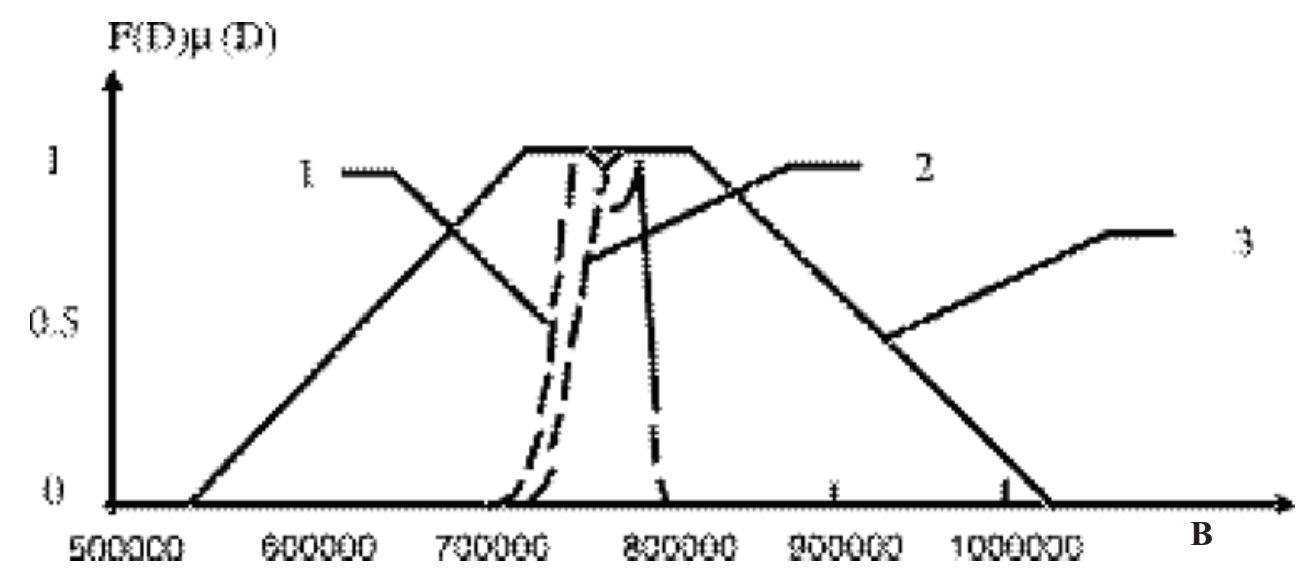

Fig. 8: Frequency distribution $F$ and fuzzy number $\mu$ for optimized benefit B

1. - Monte-Carlo method for 10,000 random steps;

2. - Monte-Carlo method for 100000000 random steps;

3. - Fuzzy approach.

It is easy to see that fuzzy approach give us some more wider fuzzy intervals then method Monte-Carlo. It is interesting that using probabilistic method. We can get even twoextreme results whereas fuzzy approach always give us the results without ambiguity. It is worth noting that probabilistic method demands too mach of random steps (about 100000000) to obtain the smooth frequency distribution of resulting benefit $\mathrm{B}$. Thus, it seems rather senseless to use this method in practice.

\section{Conclusion}

The direct numerical method for solving of fuzzy transportation problem is elaborated. The method is based on -level representation of fuzzy numbers and probability estimation of the fact that given interval is greater or equal $(>,=)$ then another interval (this idea was firstly proposed by $\mathrm{S}$. Chanas). The proposed approach makes it possible to accomplish the direct fuzzy extension of usual simplex method.

The results of case studies with using of fuzzy optimization method and Monte-Carlo method (usual linear programming with real valued but random parameters) show that the fuzzy approach have considerable advantages in comparison with Monte-Carlo method, especially from the computational point of view.

\section{References}

Bit A. K., Biswal M. P. and Alam S. S. (1992). Fuzzy programming approach to multicriteria decision making transportation problem, Fuzzy Sets and Systems 50:135-142.

Chanas S., Delgado M., Verdegay J. L. and Vila M. A. (1993). Interval and fuzzy extensions of classical transportation problems, Transportation Planning Technol. 17: 203-218.

Chanas S. and Kuchta D. (1998). Fuzzy integer transportation problem, Fuzzy sets and systems 98: 291-298.

Chanas S. and Zielinski P. (1999). Ranking fuzzy interval numbers in the setting of random sets-further results, Information Sciences 117( 3-4): 191-200.

Chanas S., Delgado M., Verdegay J. L. and Vila M. A., (1993). Rnking fuzzy interval numbers in the setting of random sets, Information sciences 69: 201-217.

Das S. K., Goswami A. and Alam S. S. (1999). Multiobjective transportation problem with interval cost, source and destination parameters, European Journal of Operational research 117: 100-112.

Dymowa L. and Dolata M. The transportation problem under probabilistic and fuzzy uncertainties, technical university of Czestochowa. 
Isermann H. (1979). The enumeration of all efficient solution for a linear multiple objective transportation problem, Naval Research Logistics Quarterly, 26 : 123-139.

Ringuest J. L. and Rinks D. B. (1987). Interactive solutions for the linear multiobjective transportation problem, European Journal of Operational Research 32: 96-106.

Sewastianow P., Rog P. and Karczewki K. (2002). A Probabilistic Method for Ordering Group of Intervals, Informatyka teoretyczna i Stosowana/Computer Science. Poliechnika Czestochowska, Rocznik 2(2): $45-53$

Sewastianow P. and Gog P. (2003). A Probability Approach to Fuzzy and Crisp Intervals Ordering, Task Quarterly 7(1): 147-156, Politechnika Czestochowska.
Sewastianow P. and Jonczyk M., Bicriterial fuzzy portfolio selection, In this issue.

Waiel F. Abd El-Wahed, (2001). A multi-objective transportation problem under fuzziness, Fuzzy Sets and System 117: 27-33.

Zimmermann H. J. (1996). fuzzy set theory and its applications. 2nd Ed. (Kluwer Academic Publishers, Dardrecht) 1991, pp 286- 296.

Received : February 24, 2010;

Accepted : September 29, 2010 